Econ. Theory 5, 295-313 (1995)

Economic
Theory

(C) Springer-Verlag 1995

\title{
Decentralizing lottery allocations in markets with indivisible commodities*
}

\author{
Rod Garratt \\ Department of Economics, University of California, Santa Barbara, CA 93106, USA
}

Received: February 3, 1993; revised version October 5, 1993

\begin{abstract}
Summary. In economies with indivisible commodities, consumers tend to prefer lotteries in commodities. A potential mechanism for satisying these preferences is unrestricted purchasing and selling of lotteries in decentralized markets, as suggested in Prescott and Townsend [Int. Econ. Rev. 25, 1-20]. However, this paper shows in several examples that such lottery equilibria do not always exist for economies with finitely many consumers. Other conditions are needed. In the examples, equilibrium and the associated welfare gains are realized if consumptions are bounded or if lotteries are based upon a common "sunspot device" as defined by Shell [mimeo, 1977] and Cass and Shell [J. Pol. Econ. 91, 193-227]. The paper shows that any lottery equilibrium is either a Walrasian equilibrium or a sunspot equilibrium, but there are Walrasian and sunspot equilibria that are not lottery equilibria.
\end{abstract}

\section{Introduction}

In economies with non-convexities, it is often the case that consumers can benefit from having their final consumption determined by lottery. This idea, which does not depend on consumers having preferences favoring risk, is demonstrated in Prescott and Townsend [18] for private information economies, and in Rogerson [19] for economies with indivisible labor. More recently, Shell and Wright [22] explore the role for lotteries and sunspots (of the type introduced by Shell [21] and Cass and Shell [3]) in markets with indivisibilities.

In this paper, the trading story of Prescott and Townsend is applied to a finite-consumer market with indivisible commodities. Economies are described for which consumers benefit by having their final consumptions determined by lottery, but such gains cannot be decentralized in a market with unrestricted purchase and

$\star$ This paper is based on Chapter 3 of my doctoral dissertation, written while I was a student at Cornell University. I thank Larry Blume, Yue Yun Chen, David Easley, Aditya Goenka, John Marshall, Bruce Smith, John Wooders and an anonymous referee. I am particularly grateful to Karl Shell and Cheng-Zhong Qin. I thank the Academic Senate at UCSB for financial support. 
sale of lotteries. That is, allowing consumer demands to take the form of individually chosen lotteries results in the non-existence of an equilibrium. It is shown, however, that equilibria for these economies exist, and welfare gains can be achieved in a decentralized manner, if specific bounds are placed on consumptions of the goods or if all consumers agree to have their final consumptions determined by the outcome of a single, commonly observed sunspot device. Equilibria of the latter type are sunspot equilibria (See Shell [21] and Cass and Shell [3]).

The analysis furthers our understanding of the relationship between three well known equilibrium concepts; Walrasian equilibrium, sunspot equilibrium and lottery equilibrium. The need for understanding along these lines was first stimulated by a claim in Prescott and Townsend [18, p. 18] that the equilibria found in their paper may be interpreted as equilibria for economies where consumers trade commodities that are contingent on some extrinsic random variable. Shell and Wright [22] launched this comparison by considering the welfare properties and existence of Walrasian equilibria and sunspot equilibria in markets with indivisible commodities. Also, Shell and Wright [22, Section 3] show that Rogerson [19] employment lotteries can be decentralized as sunspot or non-sunspot equilibrium in the continuum-of-consumers case. This paper shows that any lottery equilibrium is either a Walrasian equilibrium or a sunspot equilibrium. However, there are Walrasian and sunspot equilibria that are not lottery equilibria.

This analysis is partially motivated by the recent popularity in labor economics and macro-economics of theoretical models involving indivisibilities and lotteries. Such models have been formulated to explain observed wage differentials across occupations of different risk (Marshall [12]), cyclical fluctuations (Hansen [9]), the presence and welfare implications of unemployment (Rogerson [19], Rogerson and Wright [20]), the relationship between inflation and unemployment (Greenwood and Huffman [8]), and in the study of island economies (Prescott and Rios-Rull [16]). Cooley and Smith [4] explain why government liabilities are issued in indivisible form and Smith and Villamil [24] discuss why lotteries are used along with them. Furthermore, $\mathrm{Ng}[14]$ provides an argument why consumers facing a decision to purchase an indivisible commodity, education, might choose to purchase lottery tickets and Garratt and Marshall [7] use this notion to advance a theory of the public finance of college education.

The outline of this paper is as follows. In Section 2 the base (Walrasian) economy is described. In Section 3 that economy is viewed as a lottery economy by means of a re-specification of the consumption and production sets. A lottery equilibrium is defined and examples are given demonstrating the possibility of non-existence and how this depends on bounds placed on consumptions of the goods. In Section 4 consumers are required to make trades contingent on a commonly observed sunspot variable. In Section 5 concluding remarks are made.

\section{Trade without lotteries}

Identify individual consumers by $i=1,2, \ldots, I$. There are $j=1,2, \ldots, J$ consumption goods each of which is indivisible (i.e., individual consumption goods are only available in integer amounts). Let $\mathscr{Z}$ denote the integers and let $b_{j} \in \mathscr{Z}_{++}$denote 
the bound on the amount of consumption good $j$ that may be consumed by consumers in the economy. Thus, there is a finite number of permissible consumption bundles in the economy, each of which corresponds to a point in $\mathscr{Z}_{+}^{J}$ (the Cartesian product of $\mathscr{Z}_{+}, J$ times) with the first coordinate specifying the units of consumption good 1 and so on). Consumption bundles in the economy are described as elements of the set $C=\left\{c \in \mathscr{Z}_{+}^{J}: c_{j} \leq b_{j}, j=1, \ldots, J\right\}$ where $c_{j}$ denotes the (integer) amount of the consumption good $j$ in consumption bundle $c$. Suppose the number of consumption bundles in $C$ is equal to $K$. Denote the consumption bundles by $c^{1}, \ldots, c^{k}, \ldots, c^{K}$, and consider each consumption bundle to be a different commodity. In particular, consumption bundle $c^{k}$ is commodity $k, k=1, \ldots, K$. This method of defining commodities comes from Prescott and Townsend [17] and [18]. Quantities of the $K$ commodities are represented by points $x=\left(x^{1}, \ldots, x^{K}\right) \in \mathfrak{R}^{K}$. Thus, $\mathfrak{R}^{K}$ is the commodity space.

Without lotteries, consumers choose one of the commodities for consumption. For example, $x=(0, \ldots, 0,1,0, \ldots, 0)$. The consumption set for each consumer $i$ is

$$
X_{i}=\left\{x_{i} \in \mathfrak{R}^{K}: \sum_{k=1}^{K} x_{i}^{k}=1, \quad x_{i}^{k}=0 \text { or } 1 \forall k\right\} .
$$

The consumption bundle initially held by the consumer is the endowment commodity of which he or she has 1 unit. Let $k_{i}$ denote the endowment commodity of consumer $i$. It is assumed that $c^{k_{i}} \in C$. Let $\xi_{i} \in \mathfrak{R}^{K}$ be the endowment vector of consumer $i$. That is, $\xi_{i}=(0, \ldots, 0,1,0, \ldots, 0)$, where $\xi_{i}^{k_{i}}=1$ and $\xi_{i}^{k}=0 \quad \forall k \neq k_{i}$. Consumer preferences on bundles $c^{k}$ are given by utility functions $u_{i}\left(c^{k}\right), \mathscr{Z}^{J} \rightarrow \mathfrak{R}$, which are monotonically increasing in each of the $j$ coordinates. On $X_{i}$, this may be written equivalently as

$$
v_{i}\left(x_{i}\right)=\sum_{k=1}^{K} x_{i}^{k} u_{i}\left(c^{k}\right)
$$

Prices of commodities are given by a price vector $f=\mathfrak{R}_{+}^{K}$. The utility maximizing consumer solves

$$
\begin{gathered}
\max _{x_{i}} v_{i}\left(x_{i}\right) \\
\text { subject to } \sum_{k=1}^{K} f^{k} x_{i}^{k} \leq \sum_{k=1}^{K} f^{k} \xi_{i}^{k}=f^{k_{i}}, \\
x_{i} \in X_{i} .
\end{gathered}
$$

Given the definition of a commodity, a firm is needed to produce the commodities in the set $C$ from the endowments. The firm's production set is

$$
Z=\left\{z \in \mathscr{Z}^{K}: \sum_{k=1}^{K} \cdot z^{k} c^{k} \leq 0\right\}
$$

where $z^{k}$ is negative (respectively positive) if commodity $k$ is used as an input (respectively output) in the production plan $z$. The production process is constant returns to scale and thus there is no loss in generality in assuming a single firm. The 
profit maximization exercise of the firm is

$$
\max _{z} \sum_{k=1}^{K} f^{k} z^{k}
$$

subject to $z \in Z$.

Maximization by the firm places restrictions on the prices $f^{k}$, that are presented in the following proposition.

Proposition 1. If there exists a solution to equations (6)-(7), then there exist $\psi^{j} \geq 0$, $j=1, \ldots, J$, such that for $k=1, \ldots, K$,

$$
f^{k}=\sum_{j=1}^{l} \psi^{j} c_{j}^{k}
$$

Proof. Suppose at prices $f^{\prime}$ that $z^{\prime}$ is a solution to equations (6)-(7) but that the proposition is not true. Then at prices $f^{\prime}, z^{\prime}$ is not a solution to the less-constrained problem

$$
\begin{gathered}
\max _{z} \sum_{k=1}^{K} f^{k} z^{k} \\
\text { subject to } \sum_{k=1}^{K} z^{k} c_{j}^{k} \leq 0, j=1, \ldots, J, \\
z \in \Re^{K} .
\end{gathered}
$$

This is true, since the existence of $\psi^{j} \geq 0, j=1, \ldots, J$, that satisfy the condition of Proposition 1 , is necessary for $z^{\prime}$ to be a solution to equations (8)-(10). However, $z^{\prime}$ satisfies equations (9)-(10). Thus, there exists an alternative choice, $z^{\prime \prime}$, that satisfies equations (9)-(10) but has

$$
\sum_{k=1}^{K} f^{k \prime} z^{k \prime}>\sum_{k=1}^{K} f^{k \prime} z^{k \prime}
$$

In fact, we may choose $z^{\prime \prime}$ to be a vector of rational numbers. This is true, since $c^{k} \in \mathscr{Z}^{J}$ implies any unique solution to equations (8)-(10) contains only rational values. Also, for any non-unique solution containing irrational values, there exists an alternative solution, on the same exposed face, whose values are all rational.

If $z^{\prime \prime} \in \mathscr{Z}^{K}$ then we have a contradiction. If $z^{\prime \prime} \notin \mathscr{Z}^{K}$ then, since $c^{k} \in \mathscr{Z}^{J}$, there exists an integer $M$ such that $M z^{\prime \prime} \in \mathscr{Z}^{K}$. Clearly $M z^{\prime \prime}$ is a solution to the problem

$$
\begin{gathered}
\max _{z} \frac{1}{M} \sum_{k=1}^{K} f^{k t} z^{k} \\
\text { subject to } \\
\frac{1}{M} \sum_{k=1}^{K} z^{k} c_{j}^{k} \leq 0, j=1, \ldots, J, \\
z \in \mathscr{Z}^{K} .
\end{gathered}
$$

But this problem is equivalent to equations (6)-(7). Therefore, we again have a contradiction. 
A production plan $z \in Z$ is defined to be purely feasible (i.e., feasible in a non-stochastic sense) if

$$
z^{k}+\sum_{i=1}^{I} \xi_{i}^{k} \geq 0
$$

for all $k$. That is, the required inputs of each commodity do not exceed the total endowment of each commodity.

Definition 1. A Walrasian equilibrium (WE) is a price vector $f^{*}$ and a state $\left[\left(x_{i}^{*}\right), z^{*}\right]$ such that

(i) $x_{i}^{*}$ is a solution to equations (2)-(4) for each consumer $i$,

(ii) $z^{*}$ solves equations (6)-(7), and

(iii) $\sum_{i=1}^{I} x_{i}^{*} \leq z^{*}+\sum_{i=1}^{I} \xi_{i}$.

Since Henry [10] it has been well known that a competitive equilibrium may not exist in economies with multiple indivisible consumption goods (i.e., where the number of divisible and indivisible consumption goods is greater than two). The existence of equilibrium is also addressed for economies with some divisible goods and some indivisible goods by Broome [2] and MasColell [13]. For economies in which all consumption goods are indivisible, the type considered here, the existence of equilibrium is addressed by Dierker [5]. Dierker proves the existence of allocations which are nearly price equilibria for large economies, in the sense that the percentage of unsatisfied demand gets arbitrarily small. He also proves that existence can be assured if consumers possess a certain degree of price insensitivity.

\section{Trade with lotteries}

In order to consider lotteries, extend the consumption sets $X_{i}$ by relaxing the zero-one restrictions. The extended sets are

$$
W_{i}=\left\{w_{i} \in \mathfrak{R}_{+}^{K}: \sum_{k=1}^{K} w_{i}^{k}=1\right\} .
$$

Clearly $X_{i}$ is a subset of $W_{i}$. The idea of specifying the consumption set in this way comes from Prescott and Townsend [17] and [18]. The individual demands $w_{i} \in W_{i}$ may be interpreted as lotteries where $w_{i}^{k}$ is the probability of receiving the bundle $c^{k}$ as the final consumption. Consumer preferences over elements in their consumption sets (i.e., over lotteries) are given by functions $v_{i}\left(w_{i}\right)$ as defined in Section 2. Note that consumers are now assumed to be expected utility maximizers.

The firm also has increased production possibilities. Their production set $Z$ already contains a countable number of points, which can be denoted $z_{1}, z_{2}, \ldots z_{n}, \ldots$. In the lottery economy, the firm can also produce probability distributions over $Z$. Let $\Phi=\left\{\varphi=\left\{\varphi_{n} \geq 0\right\}_{n=1}^{\infty}: \sum_{n} \varphi_{n}=1\right\}$, where $\varphi_{n}$ denotes the probability attached 
to production plan $z_{n}$. The production set of the firm is

where $\varphi \in \Phi$.

$$
Y=\left\{y \in \mathfrak{R}^{K}: y=\sum_{n} \varphi_{n} z_{n}\right\}
$$

It is interesting to compare the production set $Y$ to that described in Prescott and Townsend [18, Section 4] for the continuum-of-consumers economy. In the latter case, inputs and outputs are defined in terms of signed measures. Thus the production set is

$$
\tilde{Y}=\left\{\tilde{y} \in L: \int c \tilde{y}(d c) \leq 0\right\}
$$

where $L$ is a linear space. For economies in which there are a finite number of commodities (i.e., consumption bundles), $k=1, \ldots, K$, the production set given by equation (18) becomes

$$
\tilde{Y}=\left\{\tilde{y} \in \mathfrak{R}^{K}: \tilde{y}=\sum_{k=1}^{K} \tilde{y}^{k} c^{k} \leq 0\right\} .
$$

As the following proposition reveals, the production sets $Y$ and $\tilde{Y}$ contain the same points (although the interpretation of the points in the two sets differ depending upon the context).

Proposition 2. $Y=\tilde{Y}$.

Proof. See the Appendix.

Because $Z \subset Y$, Proposition 1 still holds, and any equilibrium prices $f$ must be generated by shadow prices $\psi$. In view of the structure of prices, the consumer's problem is

$$
\begin{gathered}
\max _{w_{i}} v_{i}\left(w_{i}\right)=\sum_{k=1}^{K} w_{i}^{k} u_{i}\left(c^{k}\right) \\
\text { subject to } \quad \sum_{k=1}^{K} \psi \cdot c^{k} w_{i}^{k} \leq \psi \cdot c^{k_{i}}, \\
w_{i} \in W_{i} .
\end{gathered}
$$

This is a linear programming problem with two linearly independent constraints. Both this problem and the dual problem have feasible solutions, thus both have optimal solutions.

Let

$$
A_{n}=\left\{x_{n}=\left(x_{n_{1}}, \ldots, x_{n_{I}}\right) \in \mathfrak{R}^{K I}: x_{n_{i}} \in X_{i} \text { and } \sum_{i=1}^{I} x_{n_{i}} \leq z_{n}+\sum_{i=1}^{I} \xi_{i}\right\}
$$

denote the set of attainable certainty allocations corresponding to the production $z_{n} \in Z$. The sets $A_{n}$ each contain a finite number of points, which can be denoted $x_{n, 1}, \ldots, x_{n, t}, \ldots, x_{n,\left|A_{n}\right|}$. For each $n$, probability distributions over $A_{n}$ are described by

$$
\Gamma_{n}=\left\{\lambda_{n} \in \mathfrak{R}_{+}^{\left|A_{n}\right|}: \sum_{i=1}^{\left|A_{n}\right|} \lambda_{n, t}=1\right\}
$$


Definition 2. A lottery equilibrium $(L E)$ is a price vector $f^{*}$ and a state $\left[\left(w_{i}^{*}\right), \varphi^{*}\right]$ such that

(i) $w_{i}^{*}$ is a solution to equations (20)-(22) for each consumer $i$,

(ii) $y^{*}$ maximizes $f^{*} \cdot y$ subject to $y \in Y$,

(iii) for each $z_{n}$ such that $\varphi_{n}^{*}>0, z_{n}$ is purely feasible, and

(iv) there exist $\lambda_{1}^{*}, \lambda_{2}^{*}, \ldots, \lambda_{n}^{*}, \ldots$ with $\lambda_{n}^{*} \in \Gamma_{n}$ for each $n$, such that

$$
w_{i}^{k *}=\sum_{n} \varphi_{n}^{*} \sum_{\left\{t: x_{n_{i}, t}^{k}=1\right\}} \lambda_{n, t}^{*}
$$

for all $k$ and for all $i$.

Conditions (iii) and (iv) are the feasibility conditions for an equilibrium state $\left[\left(w_{i}^{*}\right), \varphi^{*}\right]$. Together, they say that individual demands $w_{i}^{k *}$ must be marginal distributions of some joint probability distribution over purely feasible allocations for the whole economy. Conditions (i)-(iii) plus $\sum_{i=1}^{I} w_{i}^{k *} \leq y^{k *}+\sum_{i=1}^{I} \xi_{i}^{k}$ for all $k$ are necessary for a LE. However, they are clearly not sufficient.

It is now possible to address the issue of existence of a LE. Existence of a LE in this model is not guaranteed. Three examples are now provided which demonstrate this. In Example 1 an economy is considered where there are no gains to having individual consumers' final consumptions determined by lottery, but where the introduction of trade involving lotteries destroys any possible equilibrium. In Example 2 an economy is considered where there are welfare improving lottery allocations that cannot be decentralized without specific bounds on the amount of each consumption good. Finally, in Example 3 the implications of having consumers in the economy who are risk loving are explored.

\subsection{Example 1}

Consider an economy with two consumers and two indivisible consumption goods. That is, commodities are consumption bundles in $\mathscr{Z}_{+}^{2}$. Let $u_{1}(c)=\left(c_{1}\right)^{2 / 3}\left(c_{2}\right)^{1 / 3}$ and $u_{2}(c)=\left(c_{1}\right)^{1 / 3}\left(c_{2}\right)^{2 / 3}$. Let $C=\left\{c \in \mathscr{Z}_{+}^{2}: c_{1} \leq 3, c_{2} \leq 3\right\}$. There are sixteen commodities. Their ordering is not important. However, it is useful to identify the following commodities: $c^{1}=(0,0), c^{2}=(1,1), c^{3}=(2,1), c^{4}=(3,1), c^{5}=(1,2), c^{6}=(3,2)$, $c^{7}=(1,3), c^{8}=(2,3), c^{9}=(3,3) .{ }^{1}$ Each consumer is endowed with one unit of commodity 2 .

Normalize prices by setting $\psi^{2}=1$. In order to rule out the possibility of an equilibrium one must show that conditions (i)-(iv) of Definition 2 are not satisfied for any $\psi^{1} \in \mathfrak{R}_{+}$. The consumer's problem does not change if we drop the zero-commodity from the set $\mathbf{C}$ and require $\sum_{k=2}^{K} w_{i}^{k} \leq 1$ for each consumer $i$. The advantage is that given our choice of preferences and endowments for each consumer we are assured that the constraint $\sum_{k=2}^{K} w_{i}^{k} \leq 1$ will never be binding and

\footnotetext{
1 These sixteen commodities are identified in the same way throughout the rest of the paper.
} 
therefore can be dropped. Then, the solution to the consumer's problem simply involves buying as much as possible of the commodity (or commodities) for which $\frac{u_{i}\left(c^{k}\right)}{\psi \cdot c^{k}}$ is the greatest. ${ }^{2}$ To reinterpret the solution as lottery the zero-commodity may $\psi \cdot c^{k}$
be reintroduced with $w_{i}^{1}=1-\sum_{k=2}^{K} w_{i}^{k}$ in the solution.

For $0 \leq \psi^{1}<.8$, consumer 1 demands commodity 4 with probability $w_{1}^{4}=$ $\frac{\psi^{1}+1}{3 \psi^{1}+1}>0$, which is not feasible. For $.8 \leq \psi^{1}<\frac{2^{4 / 3} 3^{2 / 3}-2^{2 / 3} 3}{2^{5 / 3}-2^{1 / 3} 3^{2 / 3}}$, consumer 2 demands commodity 8 with probability $w_{2}^{8}=\frac{\psi^{1}+1}{2 \psi^{1}+3}>0$, which is not feasible.

At $\psi^{1}=\frac{2^{4 / 3} 3^{2 / 3}-2^{2 / 3} 3}{2^{5 / 3}-2^{1 / 3} 3^{2 / 3}}$, consumer 2 demands any combination of commodities 8 and 5 that satisfies $\left(2 \psi^{1}+3\right) w_{2}^{8}+\left(\psi^{1}+2\right) w_{2}^{5}=\psi^{1}+1$. However, $w_{2}^{8}>0$ is not feasible. Therefore, we need only consider the case where $w_{2}^{5}=\frac{\psi^{1}+1}{\psi^{1}+2}>\frac{1}{2}$. At $\psi^{1}=\frac{2^{4 / 3} 3^{2 / 3}-2^{2 / 3} 3}{2^{5 / 3}-2^{1 / 3} 3^{2 / 3}}$, consumer 1 demands commodity 3 with probability $w_{1}^{3}=$ $\frac{\psi^{1}+1}{2 \psi^{1}+1}>\frac{1}{2}$. However, the demands $w_{2}^{5}>\frac{1}{2}$ and $w_{1}^{3}>\frac{1}{2}$ are also not feasible. To see this, note that these allocations require $y^{5}>\frac{1}{2}$ and $y^{3}>\frac{1}{2}$. But this implies $\sum_{n} \varphi_{n}^{*} z_{n}^{5}>\frac{1}{2}$ and $\sum_{n} \varphi_{n}^{*} z_{n}^{3}>\frac{1}{2}$. Given the specified endowments the pure feasibility of each $z_{n}$ for which $\varphi_{n}^{*}>0$ implies that $z_{n}^{k} \geq 0$ for all $k \neq 2$ (see Definition 2, condition (iii)). Using this fact, and since $z_{n} \in Z$, it follows that, $-2(1,1)+z_{n}^{3}(2,1)+z_{n}^{5}(1,2) \leq$ $(0,0)$ for all $z_{n}$ such that $\varphi_{n}^{*}>0$. Thus, $z_{n}^{3}$ and $z_{n}^{5}$ must each be equal to either 0 or 1 , and both cannot be equal to 1 for the same $n$. This implies $\sum_{n} \varphi_{n}^{*} \geq \sum_{n} \varphi_{n}^{*} z_{n}^{5}+$ $\sum_{n} \varphi_{n}^{*} z_{n}^{3}>1$

For $\frac{2^{4 / 3} 3^{2 / 3}-2^{2 / 3} 3}{2^{5 / 3}-2^{1 / 3} 3^{2 / 3}}<\psi^{1}<\frac{2^{5 / 3}-3^{2 / 3} 2^{1 / 3}}{3^{2 / 3} 2^{4 / 3}-2^{2 / 3} 3}$, consumer 1 demands commodity 3 with probability $w_{1}^{3}=\frac{\psi^{1}+1}{2 \psi^{1}+1}>\frac{1}{2}$ and consumer 2 demands commodity 5 with probability $w_{2}^{5}=\frac{\psi^{1}+1}{\psi^{1}+2}>\frac{1}{2}$, which is not feasible. At $\psi^{1}=\frac{2^{5 / 3}-3^{2 / 3} 2^{1 / 3}}{3^{2 / 3} 2^{4 / 3}-2^{2 / 3} 3}$, consumer 1 demands any combination of commodities 6 and 3 that satisfies $\left(3 \psi^{1}+2\right) w_{1}^{6}+$ $\left(2 \psi^{1}+1\right) w_{1}^{3}=\psi^{1}+1$. However, $w_{1}^{6}>0$ is not feasible. Therefore, we need only consider the case where $w_{1}^{3}=\frac{\psi^{1}+1}{2 \psi^{1}+1}>\frac{1}{2}$. At $\psi^{1}=\frac{2^{5 / 3}-3^{2 / 3} 2^{1 / 3}}{3^{2 / 3} 2^{4 / 3}-2^{2 / 3} 3}$, consumer 2 demands commodity 5 with probability $w_{2}^{5}=\frac{\psi^{1}+1}{\psi^{1}+2}>\frac{1}{2}$. However. we have already pointed out that the demands $w_{1}^{3}>\frac{1}{2}$ and $w_{2}^{5}>\frac{1}{2}$ are not feasible.

2 This is easily seen by looking at the dual problem. 
For $\frac{2^{5 / 3}-3^{2 / 3} 2^{1 / 3}}{3^{2 / 3} 2^{4 / 3}-2^{2 / 3} 3}<\psi^{1} \leq 1.3$, consumer 1 demands commodity 6 with probability $w_{1}^{6}=\frac{\psi^{1}+1}{3 \psi^{1}+2}>0$, which is not feasible. Finally, for $\psi^{1}>1.3$, consumer 2 demands commodity 7 with probability $w_{2}^{7}=\frac{\psi^{1}+1}{\psi^{1}+3}>0$, which is not feasible. Thus,
there does not exist a LE.

It is interesting to note that for this example there does exist an equilibrium if we assume a continuum of consumers with an equal measure (measure 1) of each type. Then, the appropriate feasibility constraint is as specified in Prescott and Townsend [18]. At prices $\psi=(1,1)$ consumer 1 demands a lottery between commodity 3 and commodity 1 with probabilities $w_{1}^{3}=\frac{2}{3}$ and $w_{1}^{1}=\frac{1}{3}$ respectively, while consumer 2 demands a lottery between commodity 5 and commodity 1 with probabilities $w_{2}^{5}=\frac{2}{3}$ and $w_{2}^{1}=\frac{1}{3}$ respectively. The production plan $y^{3}=\frac{2}{3}, y^{5}=\frac{2}{3}$, $y^{2}=-2, y^{1}=\frac{2}{3}$ and $y^{k}=0$ for all other $k$ maximizes the firm's profit at these prices. Futhermore, for these demands and production the resource constraint for the continuum-of-consumers economy is satisfied with an equality.

In Example 1 a LE does not exist for the two consumer economy. For arbitrary finite numbers of consumers a LE may or may not exist. A LE will exist at prices $\psi=(1,1)$ for all replications of the specified economy in which the number of consumers of each type is divisible by three. ${ }^{3}$

\subsection{Example 2}

Consider an economy with three consumers and two indivisible consumption goods. Let $u_{1}(c)=\left(c_{1} c_{2}\right)^{1 / 2}, u_{2}(c)=\left(c_{1}\right)^{.64}\left(c_{2}\right)^{.36}$ and $u_{3}(c)=\left(c_{1}\right)^{.36}\left(c_{1}\right)^{.64}$. Assume $C=\left\{c \in Z_{+}^{2}: c_{1} \leq 5, c_{2} \leq 5\right\}$ and that each consumer is endowed with one unit of commodity 2 . There are now thirty-six commodities. Identify the following additional commodities: $c^{10}=(4,1), c^{11}=(5,1), c^{12}=(4,2), c^{13}=(5,2), c^{14}=(5,3)$, $c^{15}=(1,4), c^{16}=(2,4), c^{17}=(5,4), c^{18}=(1,5), c^{19}=(2,5), c^{20}=(3,5), c^{21}=(4,5)$.

In this example there are welfare gains from introducing lotteries. Specifically, the allocation $w_{1}^{9}=\frac{1}{3}, w_{1}^{1}=\frac{2}{3}, w_{2}^{3}=\frac{2}{3}, w_{2}^{1}=\frac{1}{3}, w_{3}^{5}=\frac{2}{3}, w_{3}^{1}=\frac{1}{3}$, is feasible. To see this set $\varphi_{1}=\frac{1}{3}, \varphi_{2}=\frac{2}{3}$ where the pure production $z_{1}$ is given by $z_{1}^{2}=-3, z_{1}^{1}=2$, $z_{1}^{9}=1$, and the pure production $z_{2}$ is given by $z_{2}^{2}=-3, z_{2}^{1}=1, z_{2}^{3}=1, z_{2}^{5}=1$. Let $x_{1,1}$ be the point in $A_{1}$ that has $x_{1,1}^{9}=1, x_{1_{1,1}}^{k}=0$ for all $k \neq 9, x_{1_{2,1}}^{1}=x_{1_{3,1}}^{1}=1$, and $x_{1_{2}, 1}^{k}=x_{1_{3}, 1}^{k}=0$ for all $k \neq 1$. Let $x_{2,1}$ be the point in $A_{2}$ that has $x_{2_{1}, 1}^{1}=1$, $x_{2_{1,1}}^{k}=0$ for all $k \neq 1, x_{2_{2}, 1}^{3}=1, x_{2_{2}, 1}^{k}=0$ for all $k \neq 3$, and $x_{2_{3,1}}^{5}=1, x_{2_{3,1}}^{k}=0$ for all $k \neq 5$. Then, choose $\lambda_{1,1}=1$ and $\lambda_{2,1}=1$. Furthermore, $v_{i}\left(w_{i}\right)>v_{i}\left(\xi_{i}\right)$ for $i=2,3$ and $v_{1}\left(w_{1}\right)=v_{1}\left(\xi_{1}\right)$. However, we now show that this state cannot be decentralized as a LE.

Normalize prices by setting $\psi^{2}=1$. For $0 \leq \psi^{1}<.39$, consumer 2 demands commodity 11 with probability $w_{2}^{11}=\frac{\psi^{1}+1}{5 \psi^{1}+1}>0$, which is not feasible. For $.39<$

3 This sort of dependence on the number of consumers required for the existence of a competitive equilibrium also appears in the analysis of the "Bridge Game" by Shubik [17]. 
$\psi^{1}<.44$, consumer 1 demands commodity 13 with probability $w_{1}^{13}=\frac{\psi^{1}+1}{5 \psi^{1}+2}>0$, which is not feasible. For $44<\psi^{1}<\frac{4^{.64}-3^{.64}}{3^{.64} 4-4^{.64} 3}$, consumer 2 demands commodity 10 with probability $w_{2}^{10}=\frac{\psi^{1}+1}{4 \psi^{1}+1}>0$, which is not feasible.

At $\psi^{1}=\frac{4^{.64}-3^{.64}}{3^{.64} 4-4^{.64} 3}$, consumer 2 demands any combination of commodities 10 and 4 that satisfies $\left(4 \psi^{1}+1\right) w_{2}^{10}+\left(3 \psi^{1}+1\right) w_{2}^{4}=\psi^{1}+1$. However, $w_{2}^{10}>0$ is not feasible. Therefore, we need only consider the case where $w_{2}^{4}=\frac{\psi^{1}+1}{3 \psi^{1}+1}>\frac{1}{2}$. At $\psi^{1}=\frac{4^{64}-3^{.64}}{3^{.64} 4-4^{.64} 3^{6}}$, consumer 1 demands any combination of commodities 3 and 12 that satisfies $\left(2 \psi^{1}+1\right) w_{1}^{3}+\left(4 \psi^{1}+2\right) w_{1}^{12}=\psi^{1}+1$. However, $w_{1}^{12}>0$ is not feasible. Therefore, we need only consider the case where $w_{1}^{3}=\frac{\psi^{1}+1}{2 \psi^{1}+1}>\frac{1}{2}$. However, the demands $w_{2}^{4}>\frac{1}{2}$ and $w_{1}^{3}>\frac{1}{2}$ are also not feasible. For $\frac{4^{.64}-3^{.64}}{3^{.64} 4-4^{.64} 3}<\psi^{1}<$ $\frac{2^{.5} 3-5^{.5} 3^{.5}}{5^{\cdot 5} 3^{\cdot 5} 2-2^{\cdot 5} 5^{-}}$, consumer 1 demands commodity 3 with probability $w_{1}^{3}=\frac{\psi^{1}+1}{2 \psi^{1}+1}>$ $\frac{1}{2}$ and consumer 2 demands commodity 4 with probability $w_{2}^{4}=\frac{\psi^{1}+1}{3 \psi^{1}+1}>\frac{1}{2}$, which is not feasible. At $\psi^{1}=\frac{2^{\cdot 5} 3-5^{-5} 3^{.5}}{5^{5} 3^{\cdot 5} 2-2^{.5} 5}$, consumer 1 demands any combination of commodities 3 and 14 that satisfies $\left(2 \psi^{1}+1\right) w_{1}^{3}+\left(5 \psi^{1}+3\right) w_{1}^{14}=\psi^{1}+1$. However, $w_{1}^{14}>0$ is not feasible. Therefore, we need only consider the case where $w_{1}^{3}=\frac{\psi^{1}+1}{2 \psi^{1}+1}>$ $\frac{1}{2}$. At $\psi^{1}=\frac{2^{5} 3-5^{5} 3^{\cdot 5}}{5^{5} 3^{\cdot 5} 2-2^{5} 5}$, consumer 2 demands commodity 4 with probability $w_{2}^{4}=\frac{\psi^{1}+1}{3 \psi^{1}+1}>\frac{1}{2}$. However, we have already pointed out that the demands $w_{1}^{3}>\frac{1}{2}$ and $w_{2}^{4}>\frac{1}{2}$ are not feasible.

For $\frac{2^{\cdot 5} 3-5^{5} 3^{.5}}{5^{5} 3^{.5} 2-2^{.5} 5}<\psi^{1} \leq .63$, consumer 1 demands commodity 14 with probability $w_{1}^{14}=\frac{\psi^{1}+1}{5 \psi^{1}+3}>0$, which is not feasible. For $.63<\psi^{1} \leq .71$, consumer 3 demands commodity 21 with probability $w_{3}^{21}=\frac{\psi^{1}+1}{4 \psi^{1}+5}>0$, which is not feasible. For $.71<\psi^{1} \leq .79$, consumer 2 demands commodity 13 with probability $w_{2}^{13}=$ $\frac{\psi^{1}+1}{5 \psi^{1}+2}>0$, which is not feasible. For $.79<\psi^{1} \leq .89$, consumer 1 demands commodity 17 with probability $w_{1}^{17}=\frac{\psi^{1}+1}{5 \psi^{1}+4}>0$, which is not feasible. For $.89<\psi^{1} \leq 1$, consumer 3 demands commodity 20 with probability $w_{3}^{20}=\frac{\psi^{1}+1}{3 \psi^{1}+5}>0$, 
which is not feasible. For $1<\psi^{1} \leq 1.12$, consumer 2 demands commodity 14 with probability $w_{2}^{14}=\frac{\psi^{1}+1}{5 \psi^{1}+3}>0$, which is not feasible. For $1.12<\psi^{1} \leq 1.26$, consumer 1 demands commodity 21 with probability $w_{1}^{21}=\frac{\psi^{1}+1}{4 \psi^{1}+5}>0$, which is not feasible. For $1.26<\psi^{1} \leq 1.5$, consumer 3 demands commodity 19 with probability $w_{3}^{19}=$ $\frac{\psi^{1}+1}{2 \psi^{1}+5}>0$, which is not feasible. For $1.5<\psi^{1} \leq 1.59$, consumer 2 demands commodity 17 with probability $w_{2}^{17}=\frac{\psi^{1}+1}{5 \psi^{1}+4}>0$, which is not feasible. For $1.59<$ $\psi^{1}<\frac{3 \cdot{ }^{5} 5^{5} 2-2 \cdot{ }^{5} 5}{2^{5} 3-3 \cdot{ }^{5} 5^{5}}$, consumer 1 demands commodity 20 with probability $w_{1}^{20}=$ $\frac{\psi^{1}+1}{3 \psi^{1}+5}>0$, which is not feasible.

At $\psi^{1}=\frac{3 \cdot{ }^{5} 5 \cdot 52-2 \cdot{ }^{5} 5}{2 \cdot 53-3 \cdot 5^{5} \cdot{ }^{5}}$, consumer 1 demands any combination of commodities 20 and 5 that satisfies $\left(3 \psi^{1}+5\right) w_{1}^{20}+\left(\psi^{1}+2\right) w_{1}^{5}=\psi^{1}+1$. However, $w_{1}^{20}>0$ is not feasible. Therefore, we need only consider the case where $w_{1}^{5}=\frac{\psi^{1}+1}{\psi^{1}+2}>\frac{1}{2}$. At $\psi^{1}=\frac{3^{\cdot 5} 5 \cdot 52-2^{\cdot 5} 5}{2 \cdot 53-3^{\cdot 5} 5^{5}}$, consumer 3 demands commodity 7 with probability $w_{3}^{7}=\frac{\psi^{1}+1}{\psi^{1}+3}>\frac{1}{2}$. However, the demands $w_{1}^{5}>\frac{1}{2}$ and $w_{3}^{7}>\frac{1}{2}$ are also not feasible. For $\frac{3^{5} 5.52-2 .{ }^{5} 5}{2 \cdot{ }^{5} 3-3.55^{5}}<\psi^{1}<\frac{3^{.64} 4-4 \cdot{ }^{.64} 3}{4^{.64}-3.64}$, consumer 1 demands commodity 5 with probability $w_{1}^{5}=\frac{\psi^{1}+1}{\psi^{1}+2}>\frac{1}{2}$ and consumer 3 demands commodity 7 with probability $w_{3}^{7}=\frac{\psi^{1}+1}{\psi^{1}+3}>\frac{1}{2}$, which is not feasible. At $\psi^{1}=\frac{3^{\cdot 64} 4-4^{\cdot 64} 3}{4^{64}-3^{.64}}$, consumer 3 demands any combination of commodities 7 and 15 that satisfies $\left(\psi^{1}+3\right) w_{3}^{7}+$ $\left(\psi^{1}+4\right) w_{3}^{15}=\psi^{1}+1$. However, $w_{3}^{15}>0$ is not feasible. Therefore, we need only consider the case where $w_{3}^{7}=\frac{\psi^{1}+1}{\psi^{1}+3}>\frac{1}{2}$. At $\psi^{1}=\frac{3.644-4.643}{4^{4.64}-3^{.64}}$, consumer 1 demands any combination of commodities 5 and 16 that satisfies $\left(\psi^{1}+2\right) w_{1}^{5}+$ $\left(2 \psi^{1}+4\right) w_{1}^{16}=\psi^{1}+1$. However, $w_{1}^{16}>0$ is not feasible. Therefore, we need only consider the case where $w_{1}^{5}=\frac{\psi^{1}+1}{\psi^{1}+2}>\frac{1}{2}$. However, we have already pointed out that the demands $w_{3}^{7}>\frac{1}{2}$ and $w_{1}^{5}>\frac{1}{2}$ are not feasible.

For $\frac{3^{\cdot{ }^{64} 4}-4^{\cdot 64} 3}{4^{64}-3 .{ }^{64}}<\psi^{1} \leq 2.5$, consumer 3 demands commodity 15 with probability $w_{3}^{15}=\frac{\psi^{1}+1}{\psi^{1}+4}>0$, which is not feasible. For $2.5<\psi^{1} \leq 2.7$, consumer 1 demands commodity 19 with probability $w_{1}^{19}=\frac{\psi^{1}+1}{2 \psi^{1}+5}>0$, which is not feasible. 
Finally, for $\psi^{1}>2.7$, consumer 3 demands commodity 18 with probability $w_{3}^{18}=$ $\frac{\psi^{1}+1}{\psi^{1}+5}>0$, which is not feasible. Thus, there does not exist a LE.

If we set the bounds on each consumption good to 3 or 4 , then the preferred allocation can be achieved as part of a LE. Namely at prices $\psi=(1,1)$, the feasible allocation $w_{1}^{9}=\frac{1}{3}, w_{1}^{1}=\frac{2}{3}, w_{2}^{3}=\frac{2}{3}, w_{2}^{1}=\frac{1}{3}, w_{3}^{5}=\frac{2}{3}, w_{3}^{1}=\frac{1}{3}$, is utility maximizing for each consumer and the implied production maximizes profits for the firm.

\subsection{Example 3}

Consider an economy with two consumers and two indivisible consumption goods. Let $u_{1}(c)=\left(c_{1} c_{2}\right)^{3 / 4}$ and $u_{2}(c)=\left(c_{1} c_{2}\right)^{1 / 2}$. Suppose consumer 1 is endowed with 1 unit of commodity 5 and consumer 2 is endowed with 1 unit of commodity 3 . It is clear that both consumers may gain through trade over lotteries. However, for any $\psi \in \mathfrak{R}^{2}$ the solution to consumer 1's problem is for consumer 1 to demand as much probability as she can afford on the 'largest' commodity (with the desired ratio of the two consumption goods) in her consumption set. If consumers imagine they can buy commodities whose production requires more than the total resources of the economy, consumer 1's demand will not be feasible. That is, a LE will not exist.

However, if we set $b_{j}=3, j=1,2$, then a $\mathrm{LE}$ can be achieved. To show this consider prices $\psi^{*}=\left(\psi^{1}, \psi^{2}\right)=(1,1)$. Solving equations $(20)-(22)$ we find that consumer 1 wishes to spend all her income on commodity 9. This amounts to consumer 1 demanding a lottery which puts $\frac{1}{2}$ probability on commodity 9 and $\frac{1}{2}$ probability on commodity 1 . It is easily verified that this lottery also maximizes the expected utility of consumer 2 . Let $z_{1}^{3}=-1, z_{1}^{5}=-1, z_{1}^{1}=1, z_{1}^{9}=1$ and $z_{1}^{k}=0$ for all other $k$. Then $z_{1}$ is purely feasible. Also, $\varphi_{1}^{*}=1$ is profit maximizing at these prices. Let $x_{1,1}$ be the point in $A_{1}$ that has $x_{1_{1}, 1}^{1}=1, x_{1_{1}, 1}^{k}=0$ for all $k \neq 1, x_{1_{2,1}}^{9}=1$, and $x_{1_{2,1}}^{k}=0$ for all $k \neq 9$. Let $x_{1,2}$ be the point in $A_{1}$ that has $x_{1_{1}, 2}^{9}=1, x_{1_{1,2}}^{k}=0$ for all $k \neq 9$, and $x_{1_{2}, 2}^{k}=0$ for all $k \neq 1$. Then condition (iv) of Definition 2 is satisfied with $\lambda_{1,1}^{*}=\frac{1}{2}$ and $\lambda_{1,2}^{*}=\frac{1}{2}$. Thus, there exists a LE.

In this context a feasible state (i.e., a state which satisfies conditions (iii) and (iv)) $\left[\left(w_{i}\right), \varphi\right]$ is a Pareto optimum if there does not exist an alternative feasible state $\left[\left(w_{i}^{\prime}\right)_{i} \varphi^{\prime}\right]$ with the property that $v_{i}\left(w_{i}^{\prime}\right) \geq v_{i}\left(w_{i}\right)$ for all $i$ with a strict inequality for some $i$. Note that deterministic feasible states are included in this definition. Thus, states that were Pareto optimal under the previous specification of the consumption and production sets may no longer be Pareto optimal after we allow randomization. The fact that LE do not always exist suggests that in some cases there may be Pareto optimal states that cannot be decentralized as LE for any specification of endowments. This failure of the Second Welfare theorem is easily verified. In Example 1 the allocation $w_{i}^{2}=1, i=1,2$, is Pareto optimal. However, we saw that there are no prices for which both consumers will demand commodity 2 . This result does not depend on their initial endowments.

It is apparent that for a given finite-consumer economy with indivisible commodities a LE may or may not exist. Whether or not a LE exists depends on the composition of consumer characteristics in the economy and on the choice of bounds $b_{j}$. Examples 2 and 3 demonstrate that introducing specific bounds on consumptions 
of goods can result in the existence of a LE. In the next section we consider an alternative approach.

\section{Trade with a finite number of extrinsic states of nature}

Suppose that rather than being free to choose their own lotteries, consumers are only allowed to purchase state contingent commodities based on a predetermined, finite or possibly degenerate set of extrinsic states of nature. The states and their probabilities will be chosen to suit the allocation that we seek to decentralize. How members of society negotiate the selection of a commonly accepted probability space or how robust the choice of the probability space is to the introduction of alternative probability spaces is not considered.

Sunspots are introduced, in the manner of Shell and Wright [22], by means of a probability space $(S, \Sigma, \pi)$ where $S$ is the set of states, $\Sigma$ is the $\sigma$-algebra of subsets of $S$ and $\pi$ is a probability measure. If the set of states of nature is given by $S=\left\{s_{1}, \ldots s_{h}, \ldots, s_{H}\right\}$, then there are $K H$ state contingent commodities. Let $\hat{x}_{i}\left(s_{h}\right)=\left(\hat{x}_{i}^{1}\left(s_{h}\right), \ldots, \hat{x}_{i}^{k}\left(s_{h}\right), \ldots, \hat{x}_{i}^{K}\left(s_{h}\right)\right)$ and let $\hat{x}_{i}=\left(\hat{x}_{i}\left(s_{1}\right), \ldots, \hat{x}_{i}\left(s_{h}\right), \ldots, \hat{x}_{i}\left(s_{H}\right)\right)$. The consumption set of consumer $i$ is

$$
\hat{X}_{i}=\left\{\hat{x}_{i} \in \Re^{K H}: \hat{x}_{i}^{k}\left(s_{h}\right)=0 \text { or } 1 \forall k \text { and } \forall s_{h} \in S \text {, and } \sum_{k=1}^{K} \hat{x}_{i}^{k}\left(s_{h}\right)=1 \forall s_{h} \in S\right\} .
$$

Endowments do not depend on the state of nature so we let $\hat{\xi}_{i}\left(s_{h}\right)=\hat{\xi}_{i}$ for all $s_{h} \in S$. Let $p\left(s_{h}\right)=\left(p^{1}\left(s_{h}\right), \ldots, p^{k}\left(s_{h}\right), \ldots, p^{K}\left(s_{h}\right)\right)$ represent the prices of the $K$ contingent commodities in state $s_{h}$ and let $p=\left(p\left(s_{1}\right), \ldots, p\left(s_{h}\right), \ldots, p\left(s_{H}\right)\right)$.

In an economy in which there are $H$ extrinsic states of nature consumer $i$ solves

$$
\begin{gathered}
\max _{\hat{x}_{i}} \sum_{h=1}^{H} \pi\left(s_{h}\right) v_{i}\left(\hat{x}_{i}\left(s_{h}\right)\right) \\
\text { subject to } \sum_{h=1}^{H} \sum_{k=1}^{K} p^{k}\left(s_{h}\right) \hat{x}_{i}^{k}\left(s_{h}\right) \leq \sum_{h=1}^{H} p^{k_{i}}\left(s_{h}\right), \\
\hat{x}_{i} \in \hat{X}_{i}, \quad s_{h} \in S
\end{gathered}
$$

The production set of the firm is

$$
\hat{Z}=\left\{\hat{z} \in \mathscr{Z}^{K H}: \sum_{k=1}^{K} \hat{z}^{k}\left(s_{h}\right) c^{k} \leq 0, \forall s_{h} \in S\right\}
$$

The firm maximizes profits by solving

$$
\begin{gathered}
\max _{\hat{z}} \sum_{h=1}^{H} \sum_{k=1}^{K} p^{k}\left(s_{h}\right) \hat{z}^{k}\left(s_{h}\right) \\
\text { subject to } \hat{z} \in \hat{Z} .
\end{gathered}
$$

An equilibrium to the state contingent commodities economy is now defined.

Definition 3. An equilibrium to the state contingent commodities economy consists of a probability space $(S, \Sigma, \pi)$, prices $p^{*}$, allocations $\hat{x}_{i}^{*}$ and production $\hat{z}^{*}$ such that 
(i) $\hat{x}_{i}^{*}$ is a solution to equations (27)-(29) for each consumer $i$,

(ii) $\hat{z}^{*}$ is a solution to equations (31)-(32) and

(iii) $\sum_{i=1}^{I} \hat{x}_{i}^{*}\left(s_{h}\right) \leq \sum_{i=1}^{I} \widehat{\xi}_{i}+\hat{z}^{*}\left(s_{h}\right)$ for all $s_{h} \in S$.

Equilibria for which the specified state space is degenerate (contains only one state) are WE. If the specified state space is not degenerate but the allocations and production do not depend on the observed state of nature then the equilibria are non-sunspot equilibria (non-SE). If the specified state space is not degenerate and the allocation of some or all consumers or production do depend on the observed state of nature then the equilibria are sunspot equilibria (SE).

It is trivial that any non-SE may be realized as a WE although for non-convex economies it is not necessarily true that all WE will survive the introduction of extrinsic uncertainty. This was demonstrated in Shell and Wright [22] and is apparent for these economies. However, if the WE is also a LE the introduction of extrinsic uncertainty is of no consequence. This idea is the point of Proposition 3 below.

We wish to compare existence of equilibria under different trading stories for the same economy. However, a description of the economy usually includes a specification of the consumption and production sets which differ here depending on the trading story. Economies are therefore described by an array of the following base characteristics $\left(\left(u_{i}\right),\left(c^{k_{i}}\right),\left(b_{j}\right)\right)$. The specification of consumption and production sets is obvious from the equilibrium concept considered.

Proposition 3. Suppose a LE exists in which each consumer's final consumption is deterministic. Then the same state and prices are a WE for an economy with the same characteristics. Furthermore, the WE will reappear as a non-SE for any extrinsic probability space.

Proof. Let $f^{*},\left[\left(w_{i}^{*}\right), \varphi^{*}\right]$ be a deterministic LE for some economy. Suppose the deterministic production is $z_{1}$ (i.e., $\varphi_{1}^{*}=1$ ). It is obvious a WE exists at prices $f^{*}$ with $x_{i}^{*}=w_{i}^{*}$ for all $i$, and production $z^{*}=z_{1}$. Now consider any non-degenerate extrinsic probability space (one may even consider a probability space with a continuum of states (See Shell and Wright [22] for a description)). Define $\hat{x}_{i}^{*}\left(s_{h}\right)=x_{i}^{*} \forall s_{h} \in S$ and $\forall i$. Set $\hat{z}^{*}\left(s_{h}\right)=z_{1} \forall s_{h} \in S$. Let $p^{*}\left(s_{h}\right)=f^{*} \pi\left(s_{h}\right), \forall s_{h}$. Conditions (ii) and (iii) of Definition 3 are obviously satisfied. Suppose condition (i) is not. That is, suppose at prices $p^{*}\left(s_{h}\right)$ some consumer strictly prefers an allocation $\hat{x}_{i}$ in their budget set that has $\hat{x}_{i}\left(s_{h}\right) \neq \hat{x}_{i}\left(s_{h^{\prime}}\right)$ for some $s_{h}$ and $s_{h^{\prime}}$. (If a continuum of states is assumed this must occur over a set of states with strictly positive measure.) So $\sum_{h=1}^{H} \pi\left(s_{h}\right) v_{i}\left(\hat{x}_{i}\left(s_{h}\right)\right)>\sum_{h=1}^{H} \pi\left(s_{h}\right) v_{i}\left(\hat{x}_{i}^{*}\left(s_{h}\right)\right)$. Consider a lottery allocation for this consumer defined by $w_{i}=\sum_{h=1}^{H} \pi\left(s_{h}\right) \hat{x}_{i}\left(s_{h}\right)$. This consumption was affordable when $w_{i}^{*}$ was chosen. That is, since $\hat{x}_{i}$ was assumed to be affordable we have $\sum_{h=1}^{H} \sum_{k=1}^{K} p^{k}\left(s_{h}\right) \hat{x}^{k}\left(s_{h}\right) \leq$ $\sum_{h=1}^{H} p^{k_{i} *}\left(s_{h}\right)$. Also, given the specification of prices $p^{*}\left(s_{h}\right)$ and our definition of $w_{i}$ 
this implies $\sum_{k=1}^{K} f^{k *} w_{i}^{k} \leq f^{k_{i} *}$. Furthermore, $v_{i}\left(w_{i}\right)=\sum_{k=1}^{K} w_{i}^{k} u_{i}\left(c^{k}\right)=\sum_{h=1}^{H} \pi\left(s_{h}\right) v_{i}\left(\hat{x}_{i}\left(s_{h}\right)\right)$ and $v_{i}\left(w_{i}^{*}\right)=\sum_{k=1}^{K} w_{i}^{* k} u_{i}\left(c^{k}\right)=\sum_{h=1}^{H} \pi\left(s_{h}\right) v_{i}\left(\hat{x}_{i}^{*}\left(s_{h}\right)\right)$. So $v_{i}\left(w_{i}\right)>v_{i}\left(w_{i}^{*}\right)$ which is a contradiction.

Next it is shown that non-deterministic LE states can also be decentralized, using the appropriate probability space, with trade in state contingent commodities. Thus restricting the set of possible lotteries available to consumers a priori, if done correctly, does not destroy the equilibrium.

Proposition 4. Any non-deterministic LE is also a SE for an economy with the same characteristics.

Proof. Let $\left[\left(w_{i}^{*}\right), \varphi^{*}\right]$ be a LE state at prices $f^{*}$. We define a probability space $(S, \Sigma, \pi)$ as follows. Identify states in $S$ by the subscript pairs $n, t$. Let $\pi\left(s_{n, t}\right)=\varphi_{n}^{*} \lambda_{n, t}^{*}$. Clearly, $\pi\left(s_{n, t}\right) \geq 0$ and $\sum_{n, t} \pi\left(s_{n, t}\right)=1$. Define production of each commodity $k$ in each state by $\hat{z}^{k *}\left(s_{n, t}\right)=z_{n}^{k}$ and let $\hat{x}_{i}\left(s_{n, t}\right)=\pi_{n_{i}, t}, i=1, \ldots, I$, be the allocation in state $s_{n, t}$. Let prices of commodities contingent on these states be given as $p^{*}\left(s_{n, t}\right)=f^{*} \pi\left(s_{n, t}\right)$. Using these relationships it can now be shown that prices $p^{*}$, production $\hat{z}^{*}$ and allocations $\hat{x}_{i}^{*}$ constitute a $\mathrm{SE}$.

Looking at condition (i) of Definition 3 it is first of all trivially true for prices $p^{*}$ that $\hat{x}_{i}^{*}$ is in the budget set of consumer $i$. What needs to be shown is that $\hat{x}_{i}^{*}$ maximizes the expected utility of each consumer $i$ over all allocations in the budget set. Suppose not. Then for some consumer $i$ there exists an alternative allocation $\hat{x}_{i}$ in consumer $i$ 's budget set such that

$$
\sum_{n, t} \pi\left(s_{n, t}\right) v_{i}\left(\hat{x}_{i}\left(s_{n, t}\right)\right)>\sum_{n, t} \pi\left(s_{n, t}\right) v_{i}\left(\hat{x}_{i}^{*}\left(s_{n, t}\right)\right)
$$

From this relation it is possible to show that there exists a $w_{i} \in W$ satisfying the constraints of equations (20)-(22) that is preferred by consumer $i$ to $w_{i}^{*}$. For all $k$ let

$$
w_{i}^{k}=\sum_{n, t} \pi\left(s_{n, t}\right) \hat{x}_{i}^{k}\left(s_{n, t}\right)
$$

Clearly, $w_{i}^{k} \geq 0$ and $\sum_{k=1}^{K} w_{i}^{k}=1$. Thus, $w_{i} \in W$. Furthermore since $\hat{x}_{i}$ is in consumer $i$ 's budget set

$$
\sum_{n, t} \sum_{k=1}^{K} p^{k *}\left(s_{n, t}\right) \hat{x}_{i}^{k}\left(s_{n, t}\right) \leq \sum_{n, t} p^{k_{i} *}\left(s_{n, t}\right)
$$

However, given the specification of state contingent prices this means

$$
\sum_{n, t} \sum_{k=1}^{K} f^{k *} \pi\left(s_{n, t}\right) \hat{x}_{i}^{k}\left(s_{n, t}\right) \leq \sum_{n, t} f^{k_{i} *} \pi\left(s_{n, t}\right)=f^{k_{i} *}
$$

But $\sum_{n, t} \pi\left(s_{n, t}\right) \hat{x}_{i}^{k}\left(s_{n, t}\right)=w_{i}^{k}$. Thus, $\sum_{k=1}^{K} f^{k *} w_{i}^{k} \leq f^{k_{i} *}$ as required. 
The L.H.S. of equation (33) may be rewritten as

$$
\sum_{k=1}^{K} u_{i}\left(c^{k}\right) \sum_{n, t} \pi\left(s_{n, t}\right) \hat{x}_{i}^{k}\left(s_{n, t}\right)=\sum_{k=1}^{K} w_{i}^{k} u_{i}\left(c^{k}\right)
$$
By our initial specification of $\pi$ and $\left(\hat{x}_{i}^{*}\right)$, R.H.S. equals $\sum_{k=1}^{K} w_{i}^{k} * u_{i}\left(c^{k}\right)$. Thus, equation
(33) can be rewritten as

$$
\sum_{k=1}^{K} w_{i}^{k} u_{i}\left(c^{k}\right)>\sum_{k=1}^{K} w_{i}^{k *} u_{i}\left(c^{k}\right)
$$

which is a contradiction to $w_{i}^{*}$ solving equations (20)-(22) for consumer $i$. Thus, condition (i) of Definition 3 is satisfied.

It is trivial that production $\hat{z}^{*}$ is in the set $\hat{Z}$. A similar argument to that used to verify condition (i) may be used to show $\hat{z}^{*}$ is profit maximizing for the firm. Thus, condition (ii) of Definition 3 is satisfied.

Finally, note that $x_{n, t} \in A_{n}$ implies that $\sum_{i=1}^{I} x_{n_{i}, t} \leq z_{n}+\sum_{i=1}^{I} \xi_{i}$ for all $t$ and $n$. By our specification of $\hat{x}_{i}^{*}\left(s_{n, t}\right)$ and $\hat{z}^{*}\left(s_{n, t}\right)$, and since $\xi_{i}=\widehat{\xi}_{i}$, it follows that $\sum_{i=1}^{I} \hat{x}_{i}^{*}\left(s_{n, t}\right) \leq$ $\hat{z}^{*}\left(s_{n, t}\right)+\sum_{i=1}^{I} \hat{\xi}_{i}$ for all $s_{n, t} \in S$. Thus, condition (iii) of Definition 3 is satisfied.

It is thus clear that any LE is also either a WE or SE. In fact, as the following propositions illustrate, some allocations can only be decentralized if consumers are required to accept a common (possibly degenerate) randomization device.

Proposition 5. There exist economies for which WE exist that are not (deterministic) $L E$ for the same economies.

Proof. Consider the economy described in Example 1. It has been shown that a LE does not exist for this economy. However, consider prices $\psi^{*}=(1,1)$. Then autarky is a WE.

Proposition 6. There exist economies for which SE exist that are not (non-deterministic) $L E$ for the same economies.

Proof. Consider the economy described in Example 2. It has been shown that a LE does not exist for this economy. Now suppose that trade takes place in terms of state contingent claims based on three equiprobable extrinsic states of nature, $s_{1}, s_{2}$ and $s_{3}$. Then a SE does exist. Namely, prices $p^{k *}\left(s_{h}\right)=\frac{1}{3} \sum_{j=1}^{J} c_{j}^{k}$ for all $h, k$ and allocations $\hat{x}_{1}^{9}\left(s_{1}\right)=1, \hat{x}_{1}^{1}\left(s_{2}\right)=\hat{x}_{1}^{1}\left(s_{3}\right)=1, \hat{x}_{1}^{k}\left(s_{h}\right)=0$ otherwise, $\hat{x}_{2}^{3}\left(s_{2}\right)=\hat{x}_{2}^{3}\left(s_{3}\right)=1$, $\hat{x}_{2}^{1}\left(s_{1}\right)=1, \hat{x}_{2}^{k}\left(s_{h}\right)=0$ otherwise, $\hat{x}_{3}^{5}\left(s_{2}\right)=\hat{x}_{3}^{5}\left(s_{3}\right)=1, \hat{x}_{3}^{1}\left(s_{1}\right)=1, \hat{x}_{3}^{k}\left(s_{h}\right)=0$ otherwise, and production $\hat{z}^{2}\left(s_{1}\right)=-3, \hat{z}^{9}\left(s_{1}\right)=1 ; \hat{z}^{2}\left(s_{h}\right)=-3, \hat{z}^{3}\left(s_{h}\right)=1, \hat{z}^{5}\left(s_{h}\right)=1, h=2,3$; and $\hat{z}^{k}\left(s_{h}\right)=0$ otherwise, satisfy Definition 3 .

Theorem 1. Any LE is either a WE or a SE for an economy with the same characteristics. However, there are $W E$ and $S E$ that are not $L E$.

Proof. Immediate from Propositions 3, 4, 5, and 6. 
These results are analogous to results obtained by Peck and Shell [15] relating sunspot equilibria to correlated equilibria for imperfectly competitive market games economies. Peck and Shell find that a correlated equilibrium to the market game is a sunspot or non-sunspot equilibrium to the related securities game but that the converse is not necessarily true.

\section{Conclusion}

The welfare gains obtainable by having final consumptions determined by lottery cannot always be attained via decentralized equilibria if trade occurs with unrestricted purchasing and selling of lotteries. Placing bounds on quantities of consumption goods may solve the problem but this leaves open the question as to how the bounds are determined. If consumers are restricted to lotteries that are defined in terms of a common, finite set of extrinsic states of nature, a sunspot equilibrium can exist for economies that have no lottery equilibrium. In fact, sunspot-economies with a continuum of extrinsic states of nature permit no fewer equilibria than exist in lottery-economies since the state-contingent prices in the sunspot-economy may vary across states.

Nevertheless, some important questions remain unanswered. For instance, how is the probability space in the sunspot trading story selected? From a social planner's point of view this would be dictated by the particular Pareto optimal state one wishes to achieve. But this does not explain how market participants would coordinate themselves on a particular sunspot variable, or whether state contingent prices can always be found such that individuals will demand the proper allocation.

Given the difficulty of decentralizing lottery allocations, instances where consumers band together to form explicit lottery contracts are not surprising. An example in this spirit is provided by rotating savings and credit associations. Rotating savings and credit associations are organizations that pool funds enabling members to make large indivisible purchases sooner than they would be able to on their own. These organizations are studied by Besley, Coate and Loury [1] who point out that the random procedures used to allocate funds may provide higher expected utility for participants than they would get from borrowing in perfect capital markets.

\section{Appendix}

Proof of Proposition 2. The method of proof is to show that each of the sets $Y$ and $\tilde{Y}$ is equal to the convex hull of $Z$, to be denoted co $Z$.

STEP 1. (Prove that $\tilde{Y}=\operatorname{co} Z$.)

Consider the sequences of sets $\tilde{Y}^{q}=\left\{\tilde{y} \in \tilde{Y}:\left|\tilde{y}^{k}\right| \leq q, \forall k\right\}$ and $Z^{q}=$ $\left\{z_{n} \in Z:\left|z_{n}^{k}\right| \leq q, \forall k\right\}$ where $q \in \mathscr{Z}$. Each set $\tilde{Y}^{q}$ is closed, convex and bounded and $\lim _{q \rightarrow \infty} \tilde{Y}^{q}=\tilde{Y}$. Define $\operatorname{co} Z=\lim _{q \rightarrow \infty} \operatorname{co} Z^{q}$. For each $q$ the extreme points of $\tilde{Y}^{q}$ are integers which are contained in $Z^{q}$. Denote the set of these extreme points by $Z^{q^{\prime}}$. By Theorem 33 of Fenchel [6, p. 52], "a closed bounded convex set is the convex hull of its extreme points." Thus, $\tilde{Y}^{q}=C_{o} Z^{q^{\prime}}$ for all $q$. By Corollary 1.6.3 of Ichiishi 
$\left[11\right.$, p. 23] $\operatorname{co} Z^{q^{\prime}}=c o Z^{q}$. Thus, $\tilde{Y}^{q}=c o Z^{q}$ for all $q$. In the limit, as $q \rightarrow \infty$, we get $\widetilde{Y}=c o Z$.

STEP 2. (Prove that $Y=$ co $Z$.)

It is clear that $\operatorname{co} Z \subseteq Y$. It must be shown that $Y \subseteq \operatorname{co} Z$. Again let $Z^{q}=$ $\left\{z_{n} \in Z:\left|z_{n}^{k}\right| \leq q, \forall k\right\}$ where $q \in \mathscr{Z}$ and define $\operatorname{co} Z=\lim _{q \rightarrow \infty} \operatorname{co} Z^{q}$. By the definition of $Y$, it is true that for any $y \in Y$ there exists $\varphi$ such that $y=\sum_{n} \varphi_{n} z_{n}$. Let

$$
\varphi_{n}^{q}=\frac{\varphi_{n}}{\sum_{z_{n} \in Z^{q}} \varphi_{n}} .
$$

The $\lim _{q \rightarrow \infty} \varphi_{n}^{q}=\varphi_{n}$ since $\lim _{q \rightarrow \infty} \sum_{z_{n} \in Z^{q}} \varphi_{n}=1$. Let $y^{q}=\sum_{z_{n} \in Z^{q}} \varphi_{n}^{q} z_{n}$. Then $y^{q} \in \operatorname{co} Z^{q}$ and $\lim _{q \rightarrow \infty} y^{q}=y$. It must be shown that $y \in \operatorname{co} Z$. Note that $\left\{y^{q}\right\}_{q} \subseteq \operatorname{co} Z$ and $\operatorname{co} Z$ is a closed set. Therefore, $y \in c o Z$ since $y$ is the limit of a sequence contained in a closed set.

\section{References}

1. Besley, T., Coate, S., Loury, G.: The economics of rotating savings and credit associations. Amer. Econ. Assoc. 83, 792-810 (1993)

2. Broome, J.: Approximate equilibrium in economies with indivisible commodities. J. Econ. Theory 5 , 224-249 (1972)

3. Cass, D., Shell, K.: Do sunspots matter? J. Pol. Econ. 91, 193-227 (1983).

4. Cooley, T.F., Smith, B.D.: A theory of optimal denominations for government liabilities. Econ. Theory 3, 597-623 (1993)

5. Dierker, E.: Equilibrium analysis of exchange economies with indivisible commodities. Econometrica 39, 997-1008 (1971)

6. Fenchel, W.: Convex cones, sets, and functions. Mimeo, Department of Mathematics, Princeton University 1953

7. Garratt, R., Marshall, J.: Public finance of private goods: the case of higher education. J. Pol. Econ., forthcoming

8. Greenwood, J., Huffman, G.: A dynamic equilibrium model of inflation and unemployment. J. Mon. Econ. 19, 203-228 (1987)

9. Hansen, G.D.: Indivisible labor and the business cycle. J. Mon. Econ. 16, 309-327 (1985)

10. Henry, C.: Indivisibilities dan une economie d'echanges. Econometrica 38, 542-558 (1970)

11. Ichiishi, T.: In: Shell, K. (ed.) Game theory for economic analysis. New York: Academic Press 1983

12. Marshall, J.M.: Gambles and the shadow price of death. Amer. Econ. Rev. 74, 73-86 (1984)

13. Mas-Colell, A.: Indivisible commodities and general equilibrium theory. I. Econ. Theory 16, 443-456 (1977)

14. Ng, Y.K.: Why do people buy lottery tickets? Choices Involving Risk and the Indivisibility of Expenditure, J. Pol. Econ. 73, 530-535(1965)

15. Peck, J., Shell, K.: Market uncertainty: correlated and sunspot equilibria in imperfectly competitive economies. Rev. Econ. Stud. 58, 1011-1029 (1991)

16. Prescott, E., Rios-Rull, J.-V.: Classical competitive analysis of economies with islands. J. Econ. Theory 57, 73-98 (1992)

17. Prescott, E., Townsend, R.: Pareto optima and competitive equilibria with adverse selection and moral hazard. Econometrica 52, 21-45 (1984)

18. Prescott, E., Townsend, R.: General competitive analysis in an economy with private information. Int. Econ. Rev. 25, 1-20 (1984)

19. Rogerson, R.: Indivisible labor, lotteries and equilibrium. J. Mon. Econ. 21, 3-16 (1988) 
20. Rogreson, R., Wright, R.: Involuntary unemployment in economies with efficient risk sharing. J. Mon. Econ. 22, 510-515 (1988)

21. Shell, K.: Monnaie et allocation intertemporelle. Mimeo, 1977

22. Shell, K., Wright, R.: Indivisibilities, lotteries, and sunspot equilibria. Econ. Theory 2, 1-17 (1992)

23. Shubik, M.: The 'bridge game' economy: an example of indivisibilities. J. Pol. Econ. 79, 909-912 (1971)

24. Smith, B.D., Villamil, A.P.: Efficiency, randomization, and commitment in government borrowing. Mimeo, 1993

25. Townsend, R.: Arrow-Debreu programs as microfoundations of macroeconomics. In: Bewley, T. (ed.) Advances in economic theory. Cambridge: Cambridge Univerisity Press 1987 